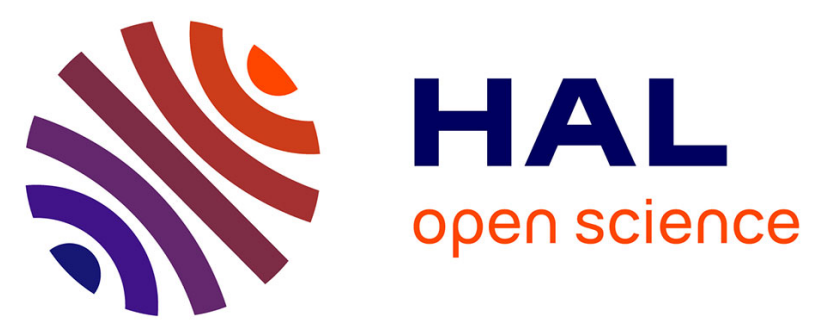

\title{
Backup State Observer Based on Optic Flow Applied to Lunar Landing
}

\author{
Guillaume Sabiron, Laurent Burlion, Grégory Jonniaux, Erwan Kervendal, \\ Eric Bornschlegl, Thibaut Raharijaona, Franck Ruffier
}

\section{- To cite this version:}

Guillaume Sabiron, Laurent Burlion, Grégory Jonniaux, Erwan Kervendal, Eric Bornschlegl, et al.. Backup State Observer Based on Optic Flow Applied to Lunar Landing. IEEE/RSJ International Conference on Intelligent Robots and Systems, Sep 2014, Chicago, United States. hal-01021322

\section{HAL Id: hal-01021322 \\ https://hal.science/hal-01021322}

Submitted on 9 Jul 2014

HAL is a multi-disciplinary open access archive for the deposit and dissemination of scientific research documents, whether they are published or not. The documents may come from teaching and research institutions in France or abroad, or from public or private research centers.
L'archive ouverte pluridisciplinaire HAL, est destinée au dépôt et à la diffusion de documents scientifiques de niveau recherche, publiés ou non, émanant des établissements d'enseignement et de recherche français ou étrangers, des laboratoires publics ou privés. 


\title{
Backup State Observer Based on Optic Flow Applied to Lunar Landing
}

\author{
Guillaume Sabiron $^{1,2}$, Laurent Burlion ${ }^{2}$, Grégory Jonniaux ${ }^{3}$, \\ Erwan Kervendal $^{3}$, Eric Bornschlegl ${ }^{4}$, Thibaut Raharijaona ${ }^{1}$, and Franck Ruffier ${ }^{1}$
}

\begin{abstract}
The observer presented in this paper, which was based on the use of three minimalistic bio-inspired Visual Motion Sensors (VMS) detecting Optic Flow (OF) cues, states was intended as a backup solution in the case of Inertial Measurement Unit (IMU) failure. Contrary to most previous Guidance Navigation and Control (GNC) solutions for planetary landing, which have involved a sensor suite including an IMU, an innovative strategy is presented here for estimating states without any need for inertial measurements, based solely on information about the relative velocity of the images of the surrounding environment. A Linear Parameter Varying (LPV) observer designed on a LPV system linearized around a reference trajectory, estimates: the ventral $\mathrm{OF}$, the expansion $\mathrm{OF}$ and the local pitch angle. A previously developed observer was applied here to a larger class of nonlinear systems by making an ingenious change of variable. Simulations performed on a lunar landing scenario yielded satisfactory performance and showed the robustness of the OF based observer to initial uncertainties and measurement noise.
\end{abstract}

\section{INTRODUCTION}

In most previous systems designed for the autonomous navigation of robotic systems, pose and attitude parameters have usually been measured or estimated during planetary landing [5], [33], [16]. However, vision based sensors and algorithms which meet the stringent weight, size and power consumption requirements of spatial applications, have recently provided new means of controlling these complex systems. Two different vision based approaches have been widely studied by performing numerical simulations:

- The first approach was based on the use of images along with information provided by sensors of other kinds or embedded knowledge of the terrain to reconstruct classical states such as velocities, attitude angles and angular velocities [19], [28], [6], [8], [27], [11]. Once these states have been estimated, classical control theory can be used to bring the system autonomously to the

\footnotetext{
* This research work is co-funded by CNRS Institutes (Life Science; Information Science; Engineering Science and Technology), the Aix-Marseille University, European Space Agency, ONERA the French Aerospace Lab and Astrium Satellites under ESA's Networking/Partnering Initiative program (NPI) for advanced technologies for space.

1 G. Sabiron, T. Raharijaona and F. Ruffier are with Aix-Marseille University, CNRS, Institute of Movement Science, Biorobotics Dept., UMR7287, 13288, Marseille, France \{Thibaut.Raharijaona, Franck.Ruffier\}euniv-amu.fr.

${ }^{2}$ G. Sabiron, and L. Burlion are with the French Aerospace Lab (ONERA, Systems Control and Flight Dynamics -DCSD-), 31055 Toulouse, France \{Guillaume.Sabiron, Laurent.Burlion\}enera.fr

3 G. Jonniaux, E. Kervendal are with Airbus Defence and Space, 31400 Toulouse, France \{Gregory. Jonniaux, Erwan.Kervendal\}@astrium.eads . net

${ }^{4}$ E. Bornschlegl is with the European Space Agency (ESTEC), 2200 AG Noordwijk, The Netherlands Eric.Bornschlegl@esa.int
}

appropriate destination. However, these recent developments are often associated with a high computational cost, mainly due to the image processing algorithm extracting visual cues from the cameras output.

- In the second approach, the system was driven on the basis of relative information extracted from images of the environment. It has been established that flying insects use the Optic Flow (OF), which provides them with relative angular velocity and proximity information with respect to obstacles, to navigate swiftly in unknown complex environments. The authors of several robotic studies inspired by insects' behavior have used the OF to perform hazardous tasks such as taking off, terrainfollowing, and landing safely and efficiently [29], [14], avoiding frontal obstacles [2], [12], [30], [4], tracking a moving target [23] and hovering and landing on a moving platform [15]. OF based lunar landing has been addressed in several studies using either a nonlinear observer connected to a LQ controller to track a constant OF reference in [32] or PID type controllers to track constant OF references [26] or exponentially decreasing [17] or more recently, Model Predictive Control [18]. In all these studies, sensors oriented at constant angles of $90^{\circ}$ and sometimes $135^{\circ}$ were used to compute specific OF expressions [26] so that:

$$
\left\{\begin{array}{l}
\omega_{x}=\frac{V_{x}}{h}=\omega_{90^{\circ}} \\
\omega_{z}=\frac{V_{z}}{h}=\omega_{90^{\circ}}-2 \omega_{135^{\circ}}
\end{array}\right.
$$

Keeping (1) constant or near a slowly varying reference trajectory while $h$ decreases ensures a soft touchdown of the closed loop system.

In other studies, OF measurements have been used as a means of estimating the usual states of the system along with other more classical sensors such as Inertial Measurement Units (IMUs), sonars, Global Positioning System (GPS), airspeed sensors and/or accelerometers [10], [22], [32], [9].

However, in all of these studies, the Inertial Measurement Unit (IMU) was the crucial cue: nowadays, an IMU is the corner stone of all the autopilots designed for vehicles of all kinds. For instance, in August 2012, the NASA scientists reported the occurrence of an IMU failure which caused the very advanced Morpheus lander prototype to crash while performing its first untethered flight [7]. IMU-less backup solutions are still urgently required in order to prevent accidents of this kind.

In the present paper, which focuses on the navigation part of the whole GNC strategy (observation issue), it is not 
proposed to address the important closed-loop control and guidance issues arising in Guidance Navigation and Control (GNC) design.

In this study (for the first time to the best of our knowledge), a novel backup solution was simulated for estimating the main parameters required to perform a bio-inspired planetary landing, namely the ventral and expansion OFs and the pitch angle [29], [26], [17] (The expansion OF has also been referred to as the inverse of the Time To Contact) in the case of IMU failure using only three strapped down OF sensors. With these hard-mounted sensors, there is no need for gimbal systems, which usually require a dedicated IMU. No additional exteroceptive or proprioceptive sensors were used in this setup. The reference landing trajectory was used to design a Linear Parameter Varying (LPV) observer. These bio-inspired lightweight, small-sized, and energy efficient sensors featuring only 6 pixels, which were previously developed and tested outdoors onboard a UAV flying freely over various fields, gave promising results in terms of the measured OF on a real life system subjected to strong disturbances [31].

In section 2, the dynamic model for the lander is described and a mathematical definition of the OF is presented. Section 3 describes the fusion scheme based on the least squares of OF measurements along with a linearized version of the model around a reference trajectory. Section 4 describes how the OF based observer was designed. Section 5 presents the results of the PANGU-based simulations. Section 6 contains some concluding comments and outlines our plans for future developments.

\section{LUNAR LANDER DYNAMICS AND OPTIC FLOW EQUATIONS}

In this section, the dynamic model of the OF system shown in Fig. 1, and the mathematical background are described. The dynamic system studied here consisted of a spacecraft, which was actuated via the main thrust $u_{t h}$ acting along $e_{3}$ the third canonical basis vector in the bodys fixed reference frame associated with the vector basis $\left(e_{1} ; e_{2} ; e_{3}\right)$ and $u_{\theta}$ creating a pitch torque. Since the lunar atmosphere was very thin, no friction or wind forces were applied to the lander. In line with previous studies in the literature, the lunar ground was assumed to be flat (with an infinite radius of curvature) (see [20]). The landers dynamic motion can be described on the vertical plane $\left(e_{x} ; e_{z}\right)$ of the inertial frame $\mathcal{I}$ associated with the vector basis $\left(e_{x} ; e_{y} ; e_{z}\right)$ by the following dynamic system:

$$
\left\{\begin{array}{c}
\dot{V}_{x}(t)=\frac{\sin (\theta(t))}{m_{l d r}(t)} u_{t h}(t) \\
\dot{V}_{z}(t)=\frac{\cos (\theta(t))}{m_{l d r}(t)} u_{t h}(t)-g_{M o o n} \\
\dot{q}(t)=\frac{R}{I} u_{\theta}(t) \\
\dot{m}_{l d r}(t)=\frac{-u_{t h}(t)}{I_{s p_{t h} .} g_{\text {Earth }}}+\frac{-\left|u_{\theta}(t)\right|}{I_{s p_{\theta} \cdot g_{\text {Earth }}}}
\end{array}\right.
$$

where $V_{x, z}$ denotes the lander's velocities in $\mathcal{I}, m_{l d r}$ stand for the lander's mass, which was assumed to be measured at all times, $\theta$ is the pitch angle (the pitch angular rate is written $q=\frac{d \theta}{d t}$ since this is taken to be a simplified 2-D problem), $t$

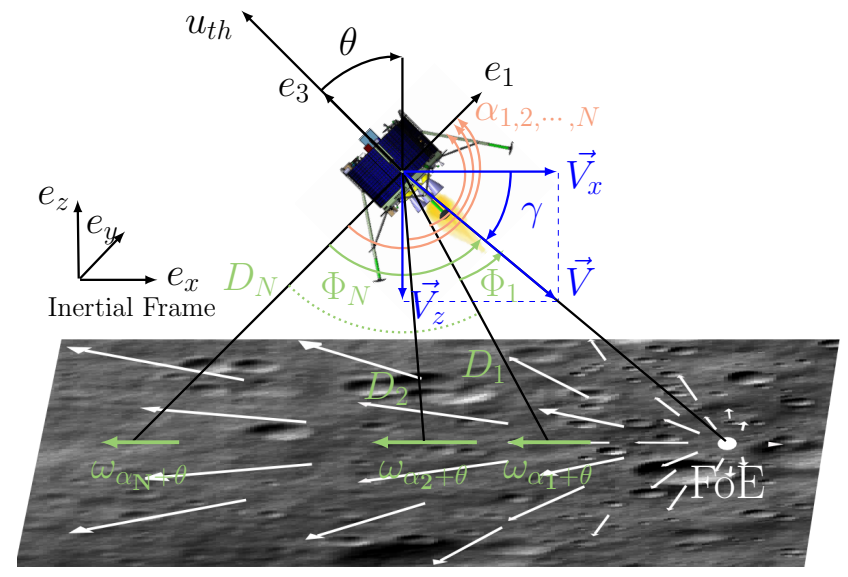

Fig. 1. Definition of the body-fixed frame $\mathcal{B}$, the inertial frame $\mathcal{I}$ and notations for the lander's dynamics and the optic flow. Example of a $\mathrm{N}$ VMS setup. Sensors fixed to the landers structure measured the OF $\omega_{\alpha_{N}+\theta}$ depending on the lander's position, velocity, pitch angle and pitch angular velocity (see (5)). It can be noted that $\Phi(t)=\alpha+\theta(t)+\gamma(t)$. The canonical vector $e_{2}$ of $\mathcal{B}$ was not included here for the sake of clarity.

denotes the time, and $g_{M o o n}$ denotes the lunar acceleration due to the gravity $\left(g_{M o o n}=1.63 \mathrm{~m} / \mathrm{s}^{2}, g_{M o o n}\right.$ was taken to be constant due to the low initial altitude). $I$ is the landers moment of inertia, and $R$ denotes the eccentricity of the attitude thrusters from the center of mass. $I_{s p}$ is the specific impulse: $I_{s p_{t h}}=325 \mathrm{~s}$ that of the braking thrusters, $I_{s p_{\theta}}=$ $287 \mathrm{~s}$ that of the attitude thrusters and $g_{\text {Earth }}=9.81 \mathrm{~m} / \mathrm{s}^{2}$ is the Earth's gravity. In the first step, we focused on a 2D setup (planar motion) in line with previous studies and because of the 6-pixel OF sensors capabilities presented in [31]. In order to progress to a 3-D setup, we would have to adopt a larger photoreceptor chip with a matrix-shaped design to be able to measure the 2-D OF involved in roll and yaw movements.

In the vertical plane, the $\mathrm{OF} \omega(\Phi)$, the sole value measured in this study, was defined in line with [24] as follows:

$$
\omega(\Phi)=\frac{V}{D} \sin (\Phi)-q
$$

where the term $\frac{V}{D} \sin (\Phi)$, which has been called the translational OF, depends on the linear velocity $V$ expressed in the inertial frame, the distance from the ground $D$ in the gaze direction and the elevation angle $\Phi$ (i.e. the angle between the gaze direction and the heading direction). In order to use the useful properties of the translational OF, a bulky gyro stabilized gimbal system has often been used to compensate for spacecrafts rotations and thus to keep the visual sensors oriented in the same direction. This means not only that the last term in (3) corresponding to the rotational OF no longer occurs in the measurements, but also that the angle $\Phi-\gamma$ was kept constant which simplifies calculations.

Although this is simple, useful mathematical framework no gimbal systems were used in this paper, it would have required an extra IMU, which is not possible since we are dealing with a case of IMU failure. The challenge of using the present strapped down sensor setup was twofold. 
First the angle $\Phi-\gamma$ was no longer constant, which ruled out simple calculations such as those performed in (1). Secondly, the rotational OF $\omega_{R}=-q$ was included in the measurements. This component $\omega_{R}$ was usually subtracted from the measured OF, $\omega_{\text {meas }}$, using IMU measurements: this operation is known as the derotation process (see [1]). Unfortunately, since the IMU was lost, it was no longer possible to perform the derotation process or to close the attitude control loop depending on pitch and angular pitch velocity measurements. We therefore used an OF based observer to estimate $\omega_{x}$ and $\omega_{z}$ during the descent as well as the pitch angle $\theta$ without using any gimbaled sensors, IMUs or velocity measurements. In the case of IMU failure, this solution could be used as a backup solution to feed control laws with accurate estimates and enable a small airborne vehicle to perform a soft landing.

\section{OPTIC FLOW FUSION AND LPV MODEL DEFINITION}

A fusion scheme was applied to the OF measurements and the landers dynamics (2) were linearized around a reference trajectory so as to obtain a Linear Parameter Varying (LPV) state space model, which was used throughout to design the observer.

Let us take only $N$ available measurements $\left(\omega_{\alpha_{1}} ; \cdots ; \omega_{\alpha_{N}}\right)$ to estimate the pitch angle $\theta$ and useful values of $\mathrm{OF}$, namely the ventral $\mathrm{OF}, \omega_{x}$, and the expansion OF, $\omega_{z}:\left[\begin{array}{l}\omega_{x}(t) \\ \omega_{z}(t)\end{array}\right]=\left[\begin{array}{c}\frac{V_{x}}{\mathrm{~h}(\mathrm{t})} \\ \frac{V_{z}}{\mathrm{~h}(\mathrm{t})}\end{array}\right]$ from the available measurements.

Assuming that we are dealing with a practically flat ground (i.e. $D=h / \cos \left(\frac{\pi}{2}-\Phi+\gamma\right)$, where $\gamma$ denotes the flight path angle (the orientation of the velocity vector with respect to the local horizontal), $h$ denotes the ground height, and $\Phi-\gamma$ denotes the angle between the gaze direction and the local horizontal) and using the notations presented on Fig. 1, we obtain the following general expression for the 2D OF:

$$
\omega_{\Phi}(t)=\frac{V(t)}{D(t)} \sin (\Phi(t))-q(t)
$$

where $\Phi(t)=\alpha+\theta(t)+\gamma(t)$, and $D=\frac{\mathrm{h}(\mathrm{t})}{\sin (\alpha+\theta(t))}$

Based on (1) this gives:

$$
\omega_{\alpha+\theta}(t)=\frac{1}{2}\left(\begin{array}{c}
1-\mathrm{c}(2(\alpha+\theta(t))) \\
\mathrm{s}(2(\alpha+\theta(t))) \\
-2
\end{array}\right)^{T}\left[\begin{array}{c}
\omega_{x}(t) \\
\omega_{z}(t) \\
q(t)
\end{array}\right]
$$

where $\sin (\gamma(\mathrm{t}))=\frac{V_{z}}{V}$ and $\cos (\gamma(\mathrm{t}))=\frac{V_{x}}{V}$ and $\alpha$, the only time invariant parameter (time notations were dropped for the sake of clarity); $\mathrm{s}(\cdot)$ and $\mathrm{c}(\cdot)$ denotes the sine function and the cosine function.

Extracting a time invariant matrix depending on $\alpha$ yields

$$
\omega_{\alpha+\theta}=\left(\begin{array}{c}
\frac{1}{2} \\
\mathrm{c}(2 \alpha) \\
\mathrm{s}(2 \alpha)
\end{array}\right)^{T}\left[\begin{array}{c}
\omega_{x}-2 q \\
-\mathrm{c}(2 \theta) \omega_{x}+\mathrm{s}(2 \theta) \omega_{z} \\
\mathrm{~s}(2 \theta) \omega_{x}+\mathrm{c}(2 \theta) \omega_{z}
\end{array}\right]
$$

The following linear system can then be solved using $N \geq$ 3 measurements if $H^{T} H$ is invertible:

$$
\left[\begin{array}{c}
\omega_{x}-2 q \\
-\cos (2 \theta) \omega_{x}+\sin (2 \theta) \omega_{z} \\
\sin (2 \theta) \omega_{x}+\cos (2 \theta) \omega_{z}
\end{array}\right]=H_{l e f t_{N}}^{-1}\left(\begin{array}{c}
\omega_{\alpha_{1}+\theta} \\
\vdots \\
\omega_{\alpha_{N}+\theta}
\end{array}\right)
$$

where the left inverse $H_{\text {left }}^{-1}$ is defined as $H_{\text {left }}^{-1}=$ $\left(H^{T} H\right)^{-1} H^{T}$, with $H_{\text {left }}^{-1} H=I_{n}, H \in M(m, n, \mathbb{R})$, $m>n$ and $H$ defined as follows:

$$
H_{N}=\left(\begin{array}{ccc}
1 & \cos \left(2 \alpha_{1}\right) & \sin \left(2 \alpha_{1}\right) \\
\vdots & \vdots & \vdots \\
1 & \cos \left(2 \alpha_{N}\right) & \sin \left(2 \alpha_{N}\right)
\end{array}\right)
$$

Remark It should be noted that for $N=3$ the matrix $H$ matrix is a square matrix:

$$
\left[\begin{array}{c}
\omega_{x}-2 q \\
-c(2 \theta) \omega_{x}+s(2 \theta) \omega_{z} \\
s(2 \theta) \omega_{x}+c(2 \theta) \omega_{z}
\end{array}\right]=H^{-1}\left(\begin{array}{c}
\omega_{\alpha_{1}+\theta} \\
\omega_{\alpha_{2}+\theta} \\
\omega_{\alpha_{3}+\theta}
\end{array}\right)
$$

with

$$
H=\left(\begin{array}{ccc}
\frac{1}{2} & \cos \left(2 \alpha_{1}\right) & \sin \left(2 \alpha_{1}\right) \\
\frac{1}{2} & \cos \left(2 \alpha_{2}\right) & \sin \left(2 \alpha_{2}\right) \\
\frac{1}{2} & \cos \left(2 \alpha_{3}\right) & \sin \left(2 \alpha_{3}\right)
\end{array}\right)
$$

It has to be checked that the following condition is satisfied to ensure that $H$ is invertible

$$
s\left(2\left(\alpha_{3}-\alpha_{2}\right)\right)+s\left(2\left(\alpha_{1}-\alpha_{3}\right)\right)+s\left(2\left(\alpha_{2}-\alpha_{1}\right)\right) \neq 0
$$

The result of the linear least squares calculation with $N>3$ (and the matrix inversion with $N=3$ ) gives a nonlinear system with three equations and four unknowns (i.e. $\omega_{x}$, $\omega_{z}, \theta$ and $q$ ) which it is impossible to solve analytically. We therefore had to make use of the knowledge available about the systems dynamics, which was done by designing an LPV observer for a linearized model of the lander's dynamics. Roughly speaking, we therefore linked together two unknowns $\theta$ and $q$, since $\dot{\theta}=q$. During space missions including entry, descent and landing phases, a reference trajectory has often been provided. This trajectory has to be followed during the actual landing to ensure the safety of the lander and reduce the fuel consumption (see [25], [34]). The reference trajectory can be expressed in terms of state trajectories, crater patterns, or a 2-D or 3-D image database [21]. In this study, we adopted a landing scenario with a computed offline reference trajectory which had to be followed. Our main assumption was that we would stay sufficiently close to this reference trajectory to be able to derive and use an LPV model for the system around the reference trajectory defined by $X^{*}=\left[h^{*}, \omega_{x}^{*}, \omega_{z}^{*}, \theta^{*}, q^{*}\right]^{T}$ involving the input control sequences $u^{*}=\left[u_{t h}^{*}, u_{\theta}^{*}\right]^{T}$ from the system dynamics (2) and the results of the linear least squares calculations (8). Linearized outputs are given by 


$$
\begin{aligned}
Y & =\left(\begin{array}{ccc}
1 & 0 & 0 \\
-\mathrm{c}\left(2 \theta^{*}\right) & \mathrm{s}\left(2 \theta^{*}\right) & 2 \omega_{x}^{*} \mathrm{~s}\left(2 \theta^{*}\right)+2 \omega_{z}^{*} \mathrm{c}\left(2 \theta^{*}\right) \\
\mathrm{s}\left(2 \theta^{*}\right) & \mathrm{c}\left(2 \theta^{*}\right) & 2 \omega_{x}^{*} \mathrm{c}\left(2 \theta^{*}\right)-2 \omega_{z}^{*} \mathrm{~s}\left(2 \theta^{*}\right)
\end{array}\right)\left(\begin{array}{c}
\Delta \omega_{x} \\
\Delta \omega_{z} \\
\Delta \theta
\end{array}\right) \\
+ & \left(\begin{array}{c}
-2 \\
0 \\
0
\end{array}\right) \Delta q+\left(\begin{array}{c}
\omega_{x}^{*}-2 q^{*} \\
-\mathrm{c}\left(2 \theta^{*}\right) \omega_{x}^{*}+\mathrm{s}\left(2 \theta^{*}\right) \omega_{z}^{*} \\
\mathrm{~s}\left(2 \theta^{*}\right) \omega_{x}^{*}+\mathrm{c}\left(2 \theta^{*}\right) \omega_{z}^{*}
\end{array}\right)
\end{aligned}
$$

Which was rewritten as follows

$$
Y=C_{1}\left(\begin{array}{c}
\Delta h \\
\Delta q
\end{array}\right)+C_{2}\left(X^{*}(t)\right)\left(\begin{array}{c}
\Delta \omega_{x} \\
\Delta \omega_{z} \\
\Delta \theta
\end{array}\right)+Y^{*}
$$

where $C_{1}=\left(\begin{array}{cc}0 & -2 \\ 0 & 0 \\ 0 & 0\end{array}\right)$.

A linearized state space model around a reference trajectory is given by

$$
\left\{\begin{array}{c}
\Delta \dot{X}=\left(\begin{array}{c}
\Delta \dot{h} \\
\Delta \dot{q} \\
\Delta \dot{\omega}_{x} \\
\Delta \dot{\omega}_{z} \\
\Delta \dot{\theta}
\end{array}\right)=A(\rho(t)) \Delta X+B(\rho(t))\left(\begin{array}{c}
\Delta u_{t h} \\
\Delta u_{\theta}
\end{array}\right) \\
\Delta Y=C(\rho(t)) \Delta X=Y-Y^{*}
\end{array}\right.
$$

with

$$
A=\left(\begin{array}{ccccc}
\omega_{z}^{*} & 0 & 0 & h^{*} & 0 \\
0 & 0 & 0 & 0 & 0 \\
\frac{-\mathrm{s}\left(\theta^{*}\right) u_{t h}^{*}}{m_{l d r} h^{*}{ }^{2}} & 0 & -\omega_{z}^{*} & -\omega_{x}^{*} & \frac{\mathrm{c}\left(\theta^{*}\right) u_{t h}^{*}}{m_{l d r} h^{*}} \\
\frac{-\mathrm{c}\left(\theta^{*}\right) u_{t h}^{*}}{m_{l d r} h^{*}}+\frac{g_{M o o n}}{h^{*}} & 0 & 0 & -2 \omega_{z}^{*} & \frac{-\mathrm{s}\left(\theta^{*}\right) u_{t h}^{*}}{m_{l d r} h^{*}} \\
0 & 1 & 0 & 0 & 0
\end{array}\right)
$$

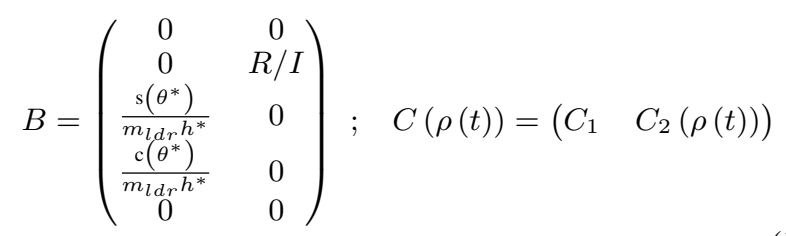

The time varying vector $\rho(t)$ depended on the reference trajectory, its associated input control signals and on the lander's mass so that $\rho(t)=\left[h^{*}, \omega_{x}^{*}, \omega_{z}^{*}, \theta^{*}, q^{*}, u_{t h}^{*}, m_{l d r}\right]^{T}$.

An LPV system was obtained; an LPV observer will now be designed to estimate state deviations from the reference trajectory, assuming that these deviations are small.

\section{OBSERVER DESIGN FOR A CLASS OF LPV SYSTEMS AND APPLICATION TO A LUNAR LANDING SCENARIO}

In this section, we present an LPV observer based on the solution proposed in [3], which was extended to include a larger class of nonlinear systems and applied to the lunar landing scenario adopted in this paper.

\section{A. LPV Observer Design}

Let us now consider an LPV system having the following form

$$
\left\{\begin{array}{c}
\dot{X}(t)=A(\rho(t)) X+B(\rho(t)) u(t) \\
y(t)=C(\rho(t)) X(t)=\left(C_{1} \quad C_{2}(\rho(t))\right) X(t)
\end{array}\right.
$$

where $X \in \mathbb{R}^{n}, \rho \in \mathbb{R}^{m}, u \in \mathbb{R}^{p}, y \in \mathbb{R}^{r}, A: \mathbb{R}^{m} \rightarrow$ $\mathcal{M}(n, n), B: \mathbb{R}^{m} \rightarrow \mathcal{M}(n, p), C: \mathbb{R}^{m} \rightarrow \mathcal{M}(r, n)$, $C_{1} \in \mathcal{M}(r, n-s), C_{2}: \mathbb{R}^{m} \rightarrow \mathcal{M}(r, s)$ where $\mathcal{M}(k, l)$ denotes the space consisting of $k \times l$ matrices with $k$ rows and $l$ columns with coefficients provided in $\mathbb{R}$. $I_{a}$ denotes the identity matrix having the size $a \times a$.

It is worth noting that the system described by (11)-(12) belongs to this class of LPV systems.

Lemma 1 Let us consider the system (15) and assume that the parameter $\rho(t)$ is measured and regularly persistent (see [3] for a definition) and the sub-matrix $C_{2}(\rho(t))$ is invertible and differentiable with respect to time, then for all gain matrices $\Theta>0$, the system:

$$
\begin{aligned}
& \dot{\hat{\bar{X}}}(t)=\bar{A} \hat{\bar{X}}(t)+\bar{B} u(t)-S^{-1} \bar{C}^{T}(\hat{y}(t)-y(t)) \\
& \dot{S}(t)=\quad-\Theta S(t)-\bar{A}(\rho(t))^{T} S(t) \\
& -S(t) \bar{A}(\rho(t))+\bar{C}(\rho(t))^{T} \bar{C}(\rho(t)) \\
& S(0)>0
\end{aligned}
$$

where

$$
\bar{X}(t)=\left(\begin{array}{cc}
I_{n-s} & 0_{n-s, s} \\
0_{s, n-s} & C_{2}(\rho(t))
\end{array}\right) X(t)=M(\rho(t)) X(t)
$$

which yields

$$
\begin{gathered}
\bar{A}=\left(\dot{M}(\rho(t)) M(\rho(t))^{-1}+M(\rho(t)) A(\rho(t)) M(\rho(t))^{-1}\right) \\
\bar{B}=M(\rho(t)) B(\rho(t)) ; \quad \bar{C}=\left(\begin{array}{ll}
C_{1} & I_{s}
\end{array}\right)
\end{gathered}
$$

is an observer for (12).

Proof: Let us now consider the new state vector resulting from the following change of variable $\bar{X}=M(\rho(t)) X$ : (12) becomes

$$
\begin{array}{ccc}
\dot{\bar{X}} & = & \left(\dot{M}(\rho(t)) M(\rho(t))^{-1}+M(\rho(t)) A(\rho(t)) M(\rho(t))^{-1}\right) \bar{X} \\
& + & M(\rho(t)) B(\rho(t)) u \\
\text { and } & &
\end{array}
$$

$$
y=\bar{C} \bar{X}
$$

which corresponds to the class of systems covered by theorem 2.1 presented in [3] (additional proof can be found in [13]).

As previous authors have pointed out, with this observer, we ensure that $\|\epsilon(t)\|^{2} \leq a e^{-\lambda_{\min }(\Theta) t}$ where $\epsilon(t)=$ $\hat{X}(t)-X(t)$ is the estimation error, $\lambda_{\min }(\Theta)$ is the smallest eigenvalue of $\Theta$ and $a$ is a constant depending on the initial errors, $\Theta$ and $u$. 


\section{B. Application to the lunar lander LPV state space model}

Applying the change of variable to the system described in (11)-(12) gives the new state vector

$$
\begin{gathered}
\Delta \bar{X}=\left(\begin{array}{c}
\Delta h \\
\Delta q \\
\Delta \bar{x}_{1} \\
\Delta \bar{x}_{2} \\
\Delta \bar{x}_{3}
\end{array}\right)=\left(\begin{array}{cc}
I_{2} & 0_{2,3} \\
0_{3,2} & C_{2}(\rho(t))
\end{array}\right) \Delta X \\
\Delta \bar{X}=M(\rho(t)) \Delta X
\end{gathered}
$$

where $0_{a, b}$ denotes the null matrix having the size $a \times b$.

The measurement equation can then be written with a time invariant observation matrix $\bar{C}$

$$
Y=Y^{*}+\left(\begin{array}{ll}
C_{1} & I_{3}
\end{array}\right) \Delta \bar{X}=Y^{*}+\bar{C} \Delta \bar{X}
$$

and the state equation can be written as follows

$$
\begin{array}{cc}
\Delta \dot{\bar{X}}= & \left(\begin{array}{cc}
I_{2} & 0_{2,3} \\
0_{3,2} & \dot{C}_{2} C_{2}^{-1}+C_{2} A C_{2}^{-1}
\end{array}\right) \Delta \bar{X} \\
+ & \left(\begin{array}{cc}
I_{2} & 0_{2,3} \\
0_{3,2} & C_{2}
\end{array}\right) B \Delta u
\end{array}
$$

with

$$
\begin{gathered}
\dot{C}_{2}=\left(\begin{array}{ccc}
0 & 0 & 0 \\
2 q^{*} \mathbf{s}\left(2 \theta^{*}\right) & 2 q^{*} \mathrm{c}\left(2 \theta^{*}\right) & C_{2_{23}} \\
2 q^{*} \mathrm{c}\left(2 \theta^{*}\right) & -2 q^{*} \mathbf{s}\left(2 \theta^{*}\right) & C_{2_{33}}
\end{array}\right) \\
C_{2_{23}}=2\left[2 q^{*} \mathbf{c}\left(2 \theta^{*}\right) \omega_{x}^{*}+\mathbf{s}\left(2 \theta^{*}\right) \dot{\omega}_{x}^{*}-2 q^{*} \mathbf{s}\left(2 \theta^{*}\right) \omega_{z}^{*}+\mathbf{c}\left(2 \theta^{*}\right) \dot{\omega}_{z}^{*}\right] \\
C_{2_{33}}=2\left[-2 q^{*} \mathbf{s}\left(2 \theta^{*}\right) \omega_{x}^{*}+\mathbf{c}\left(2 \theta^{*}\right) \dot{\omega}_{x}^{*}-2 q^{*} \mathbf{c}\left(2 \theta^{*}\right) \omega_{z}^{*}-\mathbf{s}\left(2 \theta^{*}\right) \dot{\omega}_{z}^{*}\right]
\end{gathered}
$$

By applying lemma (1), the observer can be expressed as follows:

$$
\begin{array}{cc}
\Delta \dot{\bar{X}}= & \left(\begin{array}{cc}
I_{2} & 0_{2,3} \\
0_{3,2} & \dot{C}_{2} C_{2}^{-1}+C_{2} A C_{2}^{-1}
\end{array}\right) \Delta \hat{\bar{X}} \\
+ & \left(\begin{array}{cc}
I_{2} & 0_{2,3} \\
0_{3,2} & C_{2}
\end{array}\right) B \Delta u-S^{-1} \bar{C}^{T}(\hat{Y}-Y) \\
= & \bar{A} \overline{\bar{X}}(t)+\bar{B} u(t)-S^{-1} \bar{C}^{T}(\hat{Y}(t)-Y(t)) \\
\dot{S}= & -\Theta S-\bar{A}^{T} S-S \bar{A}+\bar{C}^{T} \bar{C} ; S(0)>0
\end{array}
$$

$\Theta$ does influence the observers convergence time, but a high gain $\Theta$ is liable to amplify any measurement noise present in the simulation involving virtual images of the lunar ground. We therefore used a trial and error method and engineering knowledge of the dynamics and sensors to define the $S$ and $\Theta$ matrices as follows:

$$
S(0)=\left(\begin{array}{llll}
1 & & & \\
& 0.2 & & \\
& & I_{2} & \\
& & & 0.2
\end{array}\right) \text { and } \Theta=\left(\begin{array}{cccc}
20 & & & \\
& 0.05 & & \\
& & 20 I_{2} & \\
& & & 0.3
\end{array}\right)
$$

$\Theta$ is a tuning parameter which affects the rate of convergence of the estimated states.

\section{SIMULATION RESULTS WITH 3 OF SENSORS \\ $(N=3)$}

The results of the simulation illustrate the performances of the LPV observer. We focused here on performing simulations based on real image processing rather than using methods based on the intentional corruption of signals by adding Gaussian noise. Simulations were run under open loop conditions to obtain realistic OF measurements using three sensors placed on the lander at random angles $\alpha_{1}=$ $90^{\circ}, \alpha_{2}=120^{\circ}$ and $\alpha_{3}=160^{\circ}$ in a range corresponding to the landing scenario adopted here, so that they remained ground oriented during the entire descent phase and the condition defined by (9) was satisfied.

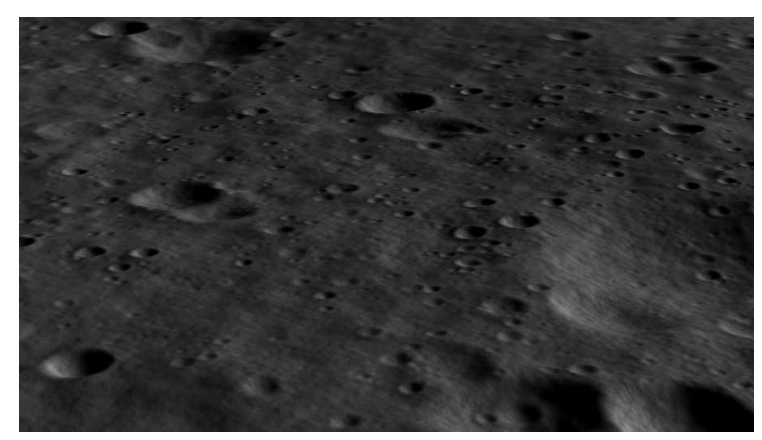

Fig. 2. Example of an image of the lunar ground obtained using PANGU software 2.70

PANGU software was used to generate images of the lunar surface, taking the position of the system, the elevation of the sun and the camera's properties into account. The simulated lunar surface was irregular and sometimes included craters up to $40 \mathrm{~m}$ deep. The images generated by PANGU contained 256 gray-scale levels and had a resolution of $256 \times 256$ pixels. Each of the OF sensors (also called VMSs) included six photoreceptors: the visual axes of each pair of photoreceptors were separated by the inter-receptor angle $\Delta \varphi=0.1^{\circ}$. The angular sensitivity of each photoreceptor obeyed a 2-D Gaussian function mimicking the angular sensitivity of the fly's photoreceptors with the acceptance angle (the angular width at half height) $\Delta \rho=\Delta \varphi=0.1^{\circ}$. These small inter-receptor and acceptance angles make it possible to compute very low velocities. Since we have such a narrow field of view, even high spatial frequency contrasts will be detected by the photodiodes, which is extremely useful at low OF levels, where fewer contrasts occur in the sensor's line of sight. In the simulated VMS model, the photoreceptors' output was simulated at each time step by convolving the PANGU-generated lunar surface image with the 2-D Gaussian filter. The simulated 6-pixel VMSs based on the actual code implemented in the sensor then assessed the OF. Fig. 2 shows a simulated image of the lunar ground generated using PANGU. One can see various craters, boulders and shadows caused by the rims of craters and the elevation of the sun. Simulations were run in open loop with precomputed input control signals using a lunar landing scenario starting with $h_{0}=1496 \mathrm{~m}, \omega_{x_{0}}=2.18^{\circ} / \mathrm{s}, \omega_{z_{0}}=$ 

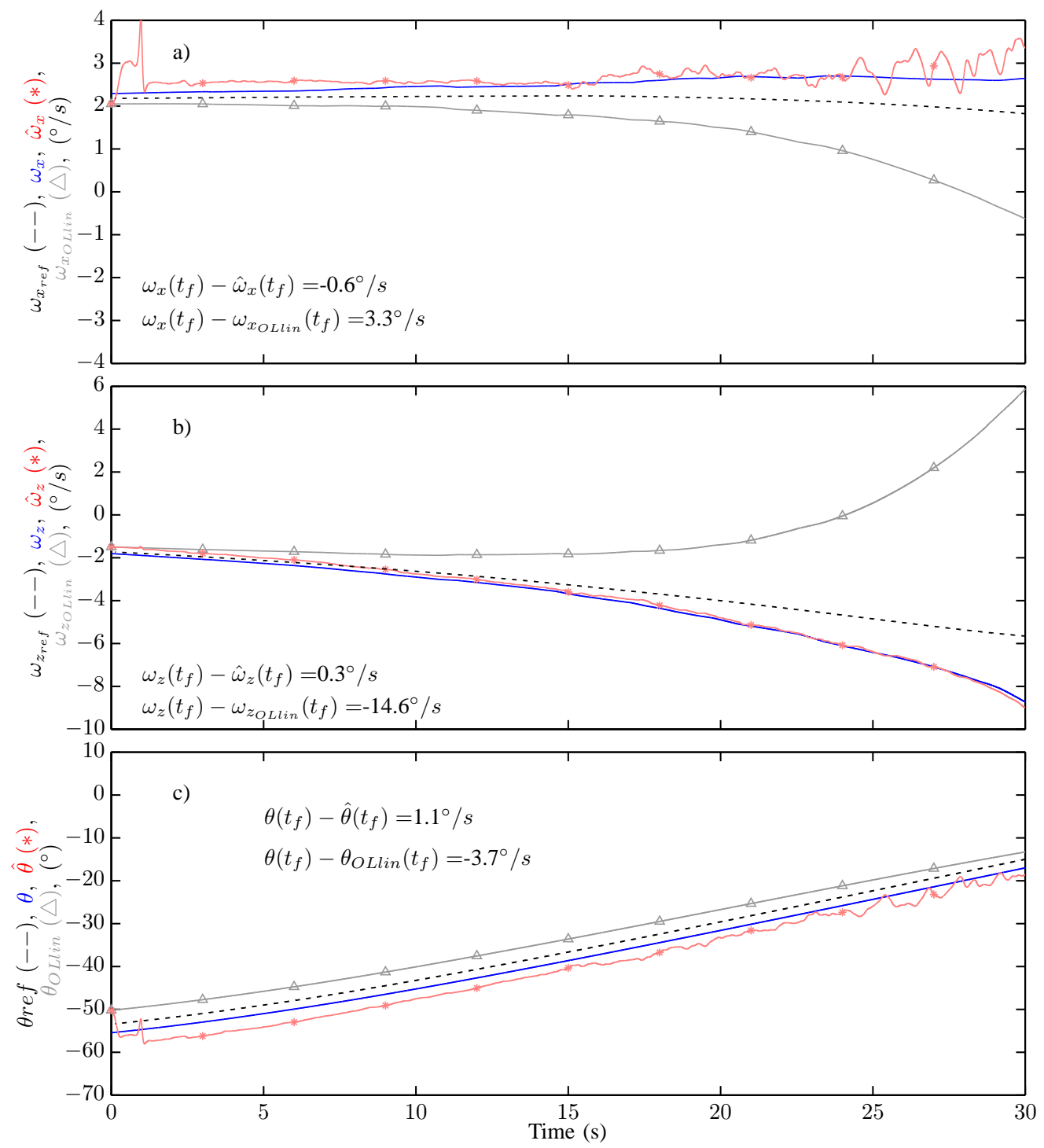

Fig. 3. Evolution of estimated $\hat{\omega}_{x}, \hat{\omega}_{x}$ and $\hat{\theta}$ in the nonlinear system with the newly designed LPV observer. The three sub-figures show the actual state of the nonlinear system (blue line), the reference state (dashed black line), the open loop linearized system ( $\triangle$-mark line) and the estimated states (*-mark line). The open loop linearized system states correspond to a simulation where the linearized system is run in open loop from the same initial conditions as the observer (states are denoted $\omega_{x_{\text {OLlin }}}, \omega_{z_{\text {OLlin }}}$ and $\theta_{O L l i n}$ ). One can see that the estimated states converged quickly toward the actual states of the nonlinear system, which were deliberately intended not to follow the reference trajectory. It should be noted in addition that the open loop linearized system alone would not have sufficed to obtain an accurate state estimation. Estimated states were initialized, giving $\Delta \hat{\omega}_{x_{0}}=-0.126^{\circ} / s$, $\Delta \hat{\omega}_{z_{0}}=0.23^{\circ} / \mathrm{s}, \Delta \hat{\theta}_{0}=3.23^{\circ}$.

$-1.72^{\circ} / \mathrm{s}, \theta_{0}=-53.43^{\circ}, q_{0}=-0.92^{\circ} / \mathrm{s}, m_{l d r_{0}}=758 \mathrm{~kg}$. Initial states deviations from the reference trajectory were chosen so that $\Delta h_{0}=100 \mathrm{~m}, \Delta \omega_{x_{0}}=-0.115^{\circ} / \mathrm{s}, \Delta \omega_{z_{0}}=$ $-0.08 / s, \Delta \theta_{0}=-2^{\circ}, \Delta q_{0}=-0.2^{\circ} / \mathrm{s}$. The estimated states were initialized, giving $\Delta \hat{h}_{0}=60 \mathrm{~m}, \Delta \hat{\omega}_{x_{0}}=-0.126^{\circ} / \mathrm{s}$, $\Delta \hat{\omega}_{z_{0}}=0.23 / \mathrm{s}, \Delta \hat{\theta}_{0}=3.23^{\circ}, \Delta \hat{q}_{0}=-0.2^{\circ} / \mathrm{s}$. Fig. 3 and Fig. 4 present simulations based on the state estimation technique presented here. In sub-figures 3.a-c, one can see the actual state (blue line), the reference state (dashed black line), the open loop linearized system $(\triangle$-mark line) and the estimated states (*-mark line). In the sub-figures 4.c, one can see the actual outputs (blue line), the reference outputs (dashed black line), the linearized open loop outputs $(\triangle$ - mark line), the estimated outputs ( $*$-mark line) and the output given by the PANGU-based measurements ( $\nabla$-mark line). The open loop linearized system states and outputs were obtained in simulation with the linearized system run in open loop from the same initial conditions as the observer (states are denoted $\omega_{x_{\text {OLlin }}}, \omega_{z_{\text {OLlin }}}, \theta_{\text {OLlin }}, h_{\text {OLlin }}, q_{O L l i n}$ and $\left.y_{O L l i n}\right)$.

As expected $\hat{\omega}_{x}, \hat{\omega}_{z}$ and $\hat{\theta}$ converged quickly toward the actual states, and it is worth noting that we also obtained a raw estimates of $h$ which took longer to converge but were not intended to be used in the future control scheme. $\hat{q}$ seems to have been more sensitive to the simulated imagebased measurements than the other estimates: this was due to the events created by the OF measurement techniques 

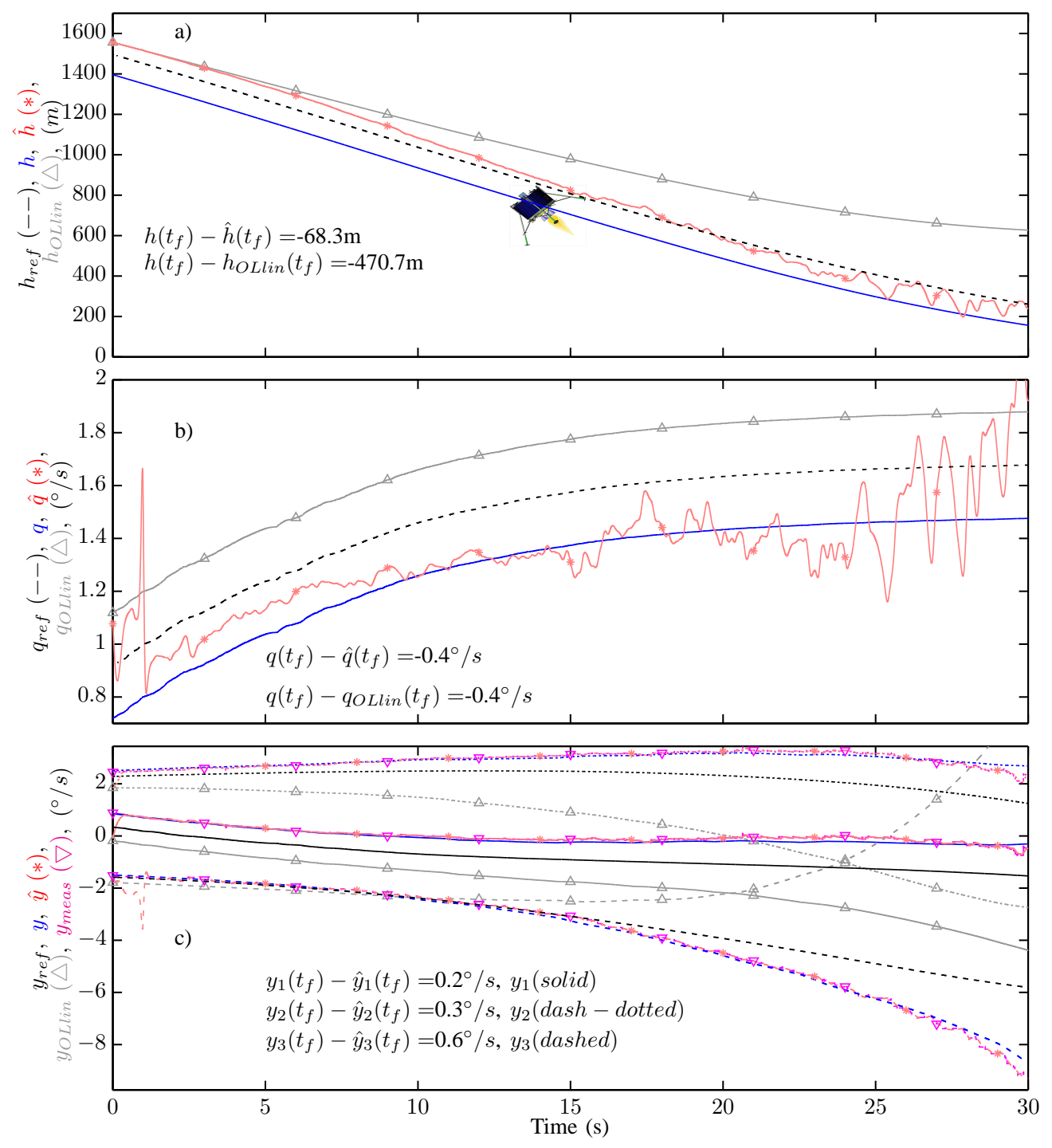

Fig. 4. Evolution of $\hat{h}, \hat{q}$ and the outputs of the observer $\hat{Y}=\left[\hat{y}_{1}, \hat{y}_{2}, \hat{y}_{3}\right]^{T}$ in the nonlinear system equipped with an LPV observer. One can see from this figure that the estimated states gave good raw estimates of the actual states despite the intrinsic sensitivity of the system to noise during the last few seconds. The estimated outputs were very close to the actual outputs of the nonlinear system. The estimated states were initialized in such a way that $\Delta \hat{h}_{0}=60 \mathrm{~m}, \Delta \hat{q}_{0}=-0.2^{\circ} / \mathrm{s}$. The open loop linearized system states correspond to a simulation where the linearized system is run in open loop from the same initial conditions as the observer (states are denoted $h_{O L l i n}, q_{O L l i n}$ and $y_{O L l i n}$ ).

as well as the noise in the measurements. Simulations with theoretical measurements were also run to check the validity of this statement (i.e. $\hat{q}-q$ tended to 0 when the outputs were perfect). Open loop linearized system ( $\triangle$-mark line) states (without the presence of an observer) did not converge toward true values, and even diverged with $\omega_{x}$ and $\omega_{z}$.

\section{CONCLUSIONS}

This study addressed the state estimation problem by using OF sensors without any need for IMU measurements. In the novel LPV observer adapted from [3] to our class of nonlinear systems by making a change of variable, the measurements used required only three lightweight bioinspired visual motion sensors hard-mounted onto the landers structure (i.e. $\omega_{\alpha_{1}+\theta}, \omega_{\alpha_{2}+\theta}$ and $\omega_{\alpha_{3}+\theta}$ ). No inertial measurements (attitude, angular velocities, angular or linear accelerations) or linear velocities or even altitudes were needed in this setup. The promising results obtained here showed the effectiveness of the observer in simulations based on software-generated images of the lunar ground. The ventral $\mathrm{OF}$, expansion $\mathrm{OF}$ and pitch angle estimates were very near the actual states although substantial sensor noise and the estimated initial states were slightly inaccurate. Due to the extreme minimalism of the present $\mathrm{OF}$ sensors and the overall backup solution, the performances of the present observer are not comparable with the high-accuracy IMU devices available. However, the present observer was intended as a backup means of driving the lander safely toward the lunar surface based on the use of small, energy efficient sensors if major sensor failures of any kind (involving not only the IMU) should occur.

The next step will consist in designing the control laws, 
taking $\omega_{x}, \omega_{z}$ and $\theta$ as inputs to bring the lander safely to the ground while following a precomputed reference trajectory. To overcome the poor estimation of the pitch angular speed, two approaches could be used:

- controlling the lander's attitude only using pitch measurement (basically a position feedback),

- or combining the estimated angular pitch rate with the time derivative of the estimated pitch angle.

It would also be interesting to compare results obtained with the present LPV observer with traditional extended unscented Kalman filters, which are designed on very similar lines and are being increasingly used to meet challenges of this kind. The LPV observer was chosen because of its wellestablished success with nonlinear systems, and also because of the proof of convergence provided by the Lyapunov theory underlying Besançon, Bornard and Hammouri's observer [3]. A cost-minimization scheme could be developed in order to improve the settings of the observers matrices $\Theta$ and $S(0)$. Further simulations will be performed in order to define the limitations of the solution proposed in terms of the initial errors and measurement noise.

\section{ACKNOWLEDGEMENTS}

We thank J. Blanc for improving the English manuscript. The authors would like to thank the three anonymous referees for their valuable comments and suggestions to improve the quality of the paper.

\section{REFERENCES}

[1] A.A. Argyros, D.P. Tsakiris, and C. Groyer. Biomimetic centering behavior [mobile robots with panoramic sensors]. Robotics Automation Magazine, 11(4):21 - 30, 68, Dec. 2004.

[2] G.L. Barrows and C. Neely. Mixed-mode VLSI optic flow sensors for in-flight control of a Micro Air Vehicle. In SPIE : Critical technologies for the future of computing, volume 4109, pages 52-63, San Diego, CA, USA, Aug. 2000.

[3] G. Besançon, G. Bornard, and H. Hammouri. Observer synthesis for a class of nonlinear control systems. European Journal of Control, 2(3):176-192, 1996.

[4] A. Beyeler, J.C. Zufferey, and D. Floreano. OptiPilot: control of takeoff and landing using optic flow. In European Micro Aerial Vehicle Conference (EMAV), volume 27, Delft, Nederlands, Sept. 2009.

[5] M. Bryson and S. Sukkarieh. Vehicle model aided inertial navigation for a uav using low-cost sensors. In Proceedings of the Australasian Conference on Robotics and Automation, 2004.

[6] T. Cheviron, T. Hamel, R. Mahony, and G. Baldwin. Robust nonlinear fusion of inertial and visual data for position, velocity and attitude estimation of uav. In Robotics and Automation, 2007 IEEE International Conference on, pages 2010-2016. IEEE, 2007.

[7] J. Devolites, J.B. Olansen, and S. Munday. Morpheus 1.5 a lander failure investigation results. In AIAA SPACE 2013 Conference and Exposition, 2013.

[8] G. Flandin, B. Polle, B. Frapard, P. Vidal, C. Philippe, and T. Voirin. Vision based navigation for planetary exploration. In 32nd Annual AAS Rocky Mountain Guidance and Control Conference, 2009.

[9] L. R. García Carrillo, A. E. Dzul López, R. Lozano, and C. Pégard. Combining stereo vision and inertial navigation system for a quadrotor uav. Journal of Intelligent \& Robotic Systems, 65(1-4):373-387, 2012.

[10] M.A. Garratt and J.S. Chahl. Vision-based terrain following for an unmanned rotorcraft. Journal of Field Robotics, 25:284-301, 2008.

[11] V. Grabe, H.H. Bulthoff, and P.R. Giordano. On-board velocity estimation and closed-loop control of a quadrotor uav based on optical flow. In Robotics and Automation (ICRA), 2012 IEEE International Conference on, pages 491-497, May 2012.
[12] S. Griffiths, J. Saunders, A. Curtis, B. Barber, T. McLain, and R. Beard. Maximizing miniature aerial vehicles. IEEE Robotics \& Automation Magazine, 13:34-43, 2006.

[13] H Hammouri and J de Leon Morales. Observer synthesis for stateaffine systems. In Decision and Control, 1990., Proceedings of the 29th IEEE Conference on, pages 784-785. IEEE, 1990.

[14] B. Hérissé, T. Hamel, R. Mahony, and F.X. Russotto. A terrainfollowing control approach for a vtol unmanned aerial vehicle using average optical flow. Autonomous Robots, 29(3-4):381-399, 2010.

[15] B. Hérissé, T. Hamel, R. Mahony, and F.X. Russotto. Landing a VTOL unmanned aerial vehicle on a moving platform using optical flow. IEEE Transactions on Robotics, 28(1):77 -89, Feb. 2012.

[16] M.D. Hua. Attitude estimation for accelerated vehicles using gps/ins measurements. Control Engineering Practice, 18(7):723-732, 2010.

[17] D. Izzo and G. de Croon. Landing with time-to-contact and ventral optic flow estimates. Journal of Guidance, Control, and Dynamics, 35 (4):1362-1367, 2011.

[18] D. Izzo and G. de Croon. Nonlinear model predictive control applied to vision-based spacecraft landing. In Proceedings of the EuroGNC 2013, 2nd CEAS Specialist Conference on Guidance, Navigation \& Control, Delft University of Technology, pages 91-107, Delft, The Netherlands, Apr. 10-12 2013.

[19] T. Jean-Marius and S.E. Strandmoe. Integrated vision and navigation for a planetary lander. Technical report, AEROSPATIAL, Espace et Défense, Les Mureaux-France. ESA, ESTEC, 1998.

[20] T. Jean-Marius and S. Trinh. Integrated vision and navigation for planetary exploration - final report. Technical Report RM-TN-00-18AS/M, Aérospatiale Espace \& Défense, 1999.

[21] A.E. Johnson and J.F. Montgomery. Overview of terrain relative navigation approaches for precise lunar landing. In Aerospace Conference, 2008 IEEE, pages 1-10. IEEE, 2008.

[22] F. Kendoul, I Fantoni, and K. Nonamib. Optic flow-based vision system for autonomous $3 \mathrm{~d}$ localization and control of small aerial vehicles. Robotics and Autonomous Systems, 57:591-602, 2009.

[23] F. Kendoul, K. Nonami, I. Fantoni, and R. Lozano. An adaptive visionbased autopilot for mini flying machines guidance, navigation and control. Autonomous Robots, 27:165-188, 2009.

[24] J.J. Koenderink and A.J. Doorn. Facts on optic flow. Biological Cybernetics, 56:247-254, 1987.

[25] X.L. Liu and G.R. Duan. Robust guidance and control of lunar lander using model reference approach. In Proc. SPIE, volume 5985, pages 856-860, 2005.

[26] V. Medici, G. Orchard, S. Ammann, G. Indiveri, and S.N. Fry. Neuromorphic computation of optic flow data bio-inspired landing using biomorphic vision sensors. Technical report, ESA, 2010.

[27] A.I. Mourikis, N. Trawny, S.I. Roumeliotis, A.E. Johnson, A. Ansar, and L. Matthies. Vision-aided inertial navigation for spacecraft entry, descent, and landing. IEEE Transactions on Robotics, 25(2):264 -280, Apr. 2009.

[28] S. Parkes, M. Dunstan, D. Matthews, I. Martin, and V. Silva. LIDARbased GNC for planetary landing: Simulation with PANGU. In R.A. Harris, editor, Data Systems in Aerospace (DASIA), page 18.1, Prague, Czech Republic, Jun. 2003.

[29] F. Ruffier and N. Franceschini. Optic flow regulation: the key to aircraft automatic guidance. Robotics and Autonomous Systems, 50:177-194, 2005.

[30] F. Ruffier and N. Franceschini. Aerial robot piloted in steep relief by optic flow sensors. In IEEE/RSJ International Conference on Intelligent Robots and Systems (IROS), pages 1266-1273. IEEE, 2008.

[31] G. Sabiron, P. Chavent, T. Raharijaona, P. Fabiani, and F. Ruffier. Low-speed optic-flow sensor onboard an unmanned helicopter flying outside over fields. In IEEE International Conference on Robotics and Automation (ICRA), 2013.

[32] F. Valette, F. Ruffier, S. Viollet, and T. Seidl. Biomimetic optic flow sensing applied to a lunar landing scenario. In International Conference on Robotics and Automation (ICRA), pages 2253-2260, 2010.

[33] J. Wendel, O. Meister, Schlaile C., and G.F. Trommer. An integrated gps/mems-imu navigation system for an autonomous helicopter. Aerospace Science and Technology, 10(6):527 - 533, 2006.

[34] J.Y. Zhou, K.L. Teo, D. Zhou, and G.H. Zhao. Optimal guidance for lunar module soft landing. Nonlinear Dynamics and Systems Theory, 10(2):189-201, 2010. 